Pathologe 2008 · [Suppl 2] 29:204-209

DOI 10.1007/s00292-008-1067-2

Online publiziert: 5. Oktober 2008

(c) Springer Medizin Verlag 2008

\author{
E. Herpel ${ }^{1,2} \cdot$ N. Koleganova $^{2} \cdot$ P. Schirmacher ${ }^{1}$ \\ ${ }^{1}$ Pathologisches Institut, Universitätsklinikum, Heidelberg \\ ${ }^{2}$ Gewebebank des Nationalen Zentrums für \\ Tumorerkrankungen, Universitätsklinik Heidelberg
}

\section{Gewebebank des Nationalen Zentrums für Tumorerkrankungen}

\section{Innovative Plattform für die translationale Tumorforschung}

Die Gewebebank des Nationalen Zentrums für Tumorerkrankungen (NCT) Heidelberg wurde im Jahre 2005 gemeinsam von der Universitätsklinik Heidelberg und dem Deutschen Krebsforschungszentrum (DKFZ) Heidelberg gegründet, um als Technologie- und Ressourcenplattform die Forschung mit humanem Gewebematerial, insbesondere Tumorgewebe, zu unterstützen. Die Gewebebank war damit national die erste Einrichtung ihrer Art, die im Kontext eines „Comprehensive Cancer Center" (CCC) eingerichtet wurde. Die Gewebebank wird als Sektion des NCT vom Pathologischen Institut der Universitätsklinik Heidelberg betrieben (• Abb. 1). Hierbei wurde von Beginn an ein umfassendes Konzept angestrebt, das im Einzelnen folgende Zielsetzungen verfolgte:

- standortumfassende und ausschließlich der Forschung dienende NonProfit-Einrichtung, die unter „good scientific practice“ Gesichtspunkten besonders die translationale Forschung und das NCT-Konzept unterstützt,

- Förderung einer integrierten, interdisziplinären und umfassenden Gewebebankeinrichtung als CCCModellstruktur,

- Pro- und retrospektive Gewebesammlung einschließlich Erstellung geeigneter Kollektive,
- Schaffung eines umfassenden, ethisch-rechtlich geprüften und akzeptierten Rahmens,

- Aufbau und Weiterentwicklung gewebebankassoziierter innovativer Techniken,

- Akzeptanz von klinischen und morphologischen Daten als wesentliche Grundlage wissenschaftlicher Arbeit,

- Einrichtung eines umfassenden Qualitätsmanagements.

Auf die Umsetzung und Fortentwicklung der einzelnen Gesichtspunkte wird nachfolgend im Einzelnen eingegangen.

\section{Standortumfassende translationale Tumorforschung}

Die Heidelberger Gewebebank wird vom DKFZ Heidelberg und der Universitätsklinik Heidelberg finanziert und nimmt für ihre Leistungen in aller Regel kein Entgelt. In besonderen Situationen ist sie jedoch berechtigt, ein angemessenes Nutzungsentgelt $\mathrm{zu}$ erheben. Sie ist grundsätzlich eine Non-Profit-Einrichtung und zur ausschließlich zweckbezogenen Verwendung ihrer Mittel verpflichtet. Ein besonderer Auftrag der Gewebebank ist es, zur Weiterentwicklung des CCC-Konzeptes im Rahmen des NCT Heidelberg aktiv beizutragen und insbesondere Pro- jekte der translationalen Tumorforschung zu fördern.

Die Gewebebank sichert die umfassende Versorgung des Standortes Heidelberg mit humanem Gewebe für die biomedizinische Forschung. Alle wesentlichen operierenden Einheiten tragen durch Gewebeeinsendungen und antragsbezogene Bereitstellung klinischer und Follow-up-Daten zur Gewebebank bei. Die Abteilung Allgemeine Pathologie und Pathologische Anatomie des Pathologischen Instituts der Universitätsklinik Heidelberg betreibt die Gewebebank und stellt projektbezogen die erforderlichen histopathologischen Daten zur Verfügung. Antragsberechtigt an die Gewebebank sind ausschließlich angestellte Wissenschaftler der die Gewebebank tragenden Einrichtungen. Die Durchführung von Kooperationsprojekten mit externen Partnern oder auch kommerziellen Einrichtungen ist jedoch möglich.

Eine Besonderheit der Gewebebank ist, dass sie ihren Schwerpunkt nicht auf das „reine Sammeln“ von Geweben, sondern auf die gezielte Projektarbeit legt. Es ist ihr ausdrückliches Ziel, Forschung zu ermöglichen und zu befördern. Dies wird besonders durch das Instrument der Projektbetreuung gewährleistet: Nach Einreichung eines kurzen, maximal halbseitigen Antrages wird von der Gewebebank in direktem Kontakt mit dem Projektleiter Art, 
erforderliche Zahl und Asservierungsform der Gewebe ermittelt. Die Möglichkeit zur Realisierung des Projektes wird individuell geprüft. Ist das Projekt in Bezug auf die vorliegenden Gewebe realisierbar, wird die entsprechende klinische Einheit um ein Votum und im positiven Fall die Benennung eines klinischen Projektbetreuers gebeten; von der Gewebebank wird zudem ein pathohistologischer Projektbetreuer zugeordnet, bei dem es sich in der Regel um einen erfahrenen Histopathologen mit entsprechendem Forschungshintergrund handelt. Bei multilateralen Projekten und in Konfliktfällen werden der Beirat und alle betroffenen klinischen Einheiten befragt.

Diese Bearbeitungsform hat sich als praktikabel und hocheffizient erwiesen; es lässt sich eine hohe klinische Akzep$\tan z$ erzielen. Bei über 200 betreuten Forschungsprojekten konnte die Gewebebank Heidelberg eine Projekterfüllungsquote von über $98 \%$ erreichen. Seltene Ablehnungsgründe waren Interferenz mit der pathohistologischen Diagnostik bzw. Noncompliance des beantragenden Projektleiters mit resultierendem Veto des Beirats.

\section{Gewebebank als CCC-Modell}

Von Anfang an war es Ziel der Betreiber, eine Gewebebank mit Modellcharakter zu schaffen. Es wurde daher besonderen Wert darauf gelegt, die Strukturen, Regularien und Fortschritte der Gewebebank publik und transparent zu machen. Hierzu wurde die Gewebebank in die Arbeitsgemeinschaft (AG) der Comprehensive Cancer Centers (CCCs) Deutschland integriert. Auf Betreiben der Heidelberger Gewebebank wurde in 2006 eine Interessengemeinschaft der Gewebebanken der CCCs und Tumorzentren gegründet. Diese hält regelmäßig in halbjährlichem Abstand Arbeitstreffen ab, an denen Vertreter von etwa 15 Standorten teilnehmen. Bei diesen Arbeitstreffen wird über die jeweiligen Fortschritte und Problemfelder berichtet, es erfolgt ein intensiver Informationsaustausch über alle Fragen der Gewebebank und gemeinsame Initiativen werden gestartet. Die Interessengemeinschaft berichtet zudem regelmäßig an die AG der CCCs. Durch

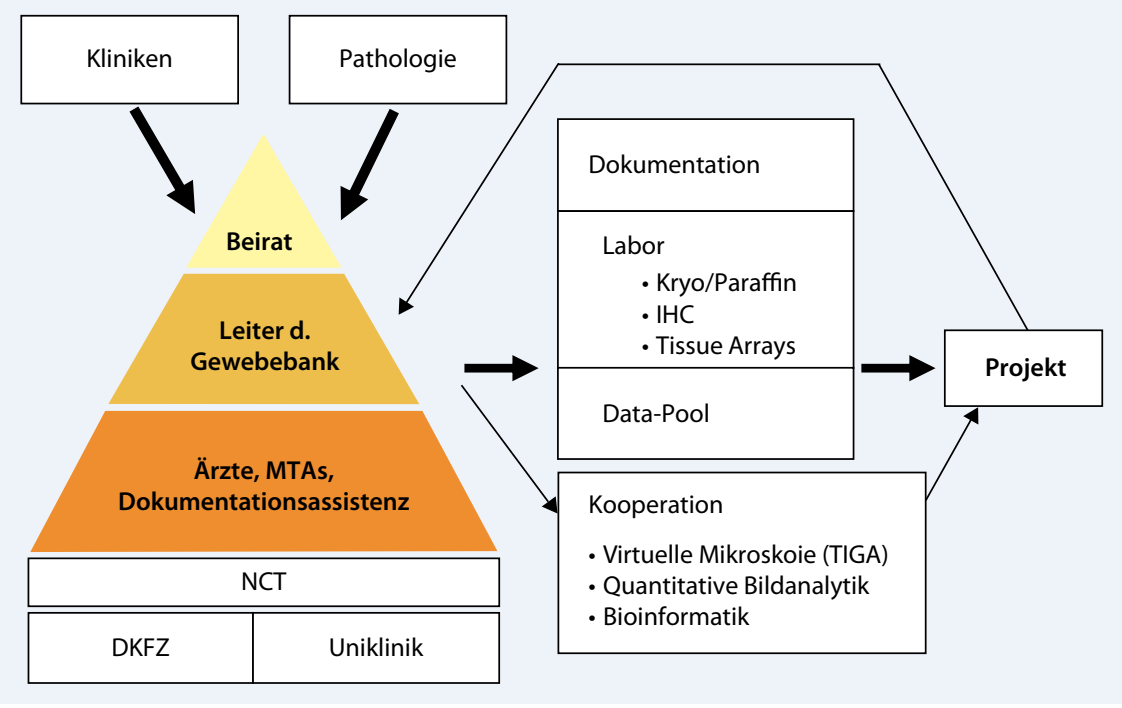

Abb. $1 \Delta$ Struktur der Gewebebank des NCT Heidelberg

Unterstützung der Interessengemeinschaft konnten bereits an vielen Standorten die Einrichtung vergleichbarer Gewebebankstrukturen unterstützt und praktische Probleme gelöst werden. Dies hat auch die Gewebebankstrukturen im Rahmen von CCCs und entsprechende Fördermittelbeantragungen nicht unerheblich geprägt.

Als wesentliches Instrument der Kontaktaufnahme und des Informationsaustauscheshatsich mittlerweiledieHomepage der Gewebebank entwickelt (gewebebank. nct@med.uni-heidelberg.de).

\section{Pro- und retrospektive Gewebesammlung}

Eine wesentliche Aufgabe der Gewebebank ist das effiziente Sammeln, Erfassen und Archivieren von Geweben. Dieses umfasst sowohl das prospektive Sammeln von Frischgeweben als auch die Verwendung von Paraffingeweben. Diese Funktionen werden in erheblichem Maß durch Standortgegebenheiten (z. B. Kooperation und Operationszahlen klinischer Einheiten, personelle Ressourcen, Forschungsbedarf, Datenbanken und Archivierungsformat) unterstützt. Aus der Erfahrung der Heidelberger Gewebebank ergeben sich jedoch eine Reihe verallgemeinerbare Erkenntnisse:

- Unter Einbindung lokal präexistenter Transportstrukturen und Lokalisation müssen Abläufe eingerichtet werden, die ein zeitnahes Einfrieren ermögli- chen und eine möglichst hohe Frischgewebsasservierungsquote gewährleisten. Hierfür können dezentrale Standorte sinnvoll sein.

- Die ausreichende und angemessene Form der Frischgewebsarchivierung ist eine noch nicht abschließend geklärte Frage, die durch Kostengesichtspunkte und wissenschaftliche Erfordernisse beeinflusst wird. In Heidelberg erfolgt die Asservierung bei $-80^{\circ} \mathrm{C}$, was bislang nicht projektlimitierend war.

- Es ist eine allgemeine Erfahrung, dass eine erhebliche Zahl beantragter Projekte durchaus gleichwertig auch mit Paraffingewebe/"multi-tissuearray" (MTA) vollwertig erfüllt werden kann (,downscaling“). Häufig, so auch in Heidelberg, existieren umfangreiche präexistente Frischgewebssammlungen. Unter der Maßgabe, dass sie nicht mit der SOP (,standard operating procedures") -gesteuerten prospektiven Frischgewebsasservierung vermischt werden und eine entsprechende histologische Ausgangskontrolle erfolgt, sind diese Sammlungen durchaus wertvoll; die Gewebequalität kann erstaunlich gut sein; ihre Übernahme in das Gewebebankkonzept ist zwar aufwendig, sollte aber geprüft werden, um eine rasche Projektbetreuung zu erleichtern und insbesondere auch seltenere Entitäten in ausreichender Menge vorhalten zu können. Insbesondere 


\section{Hauptreferate: Biobanking in der Pathologie}

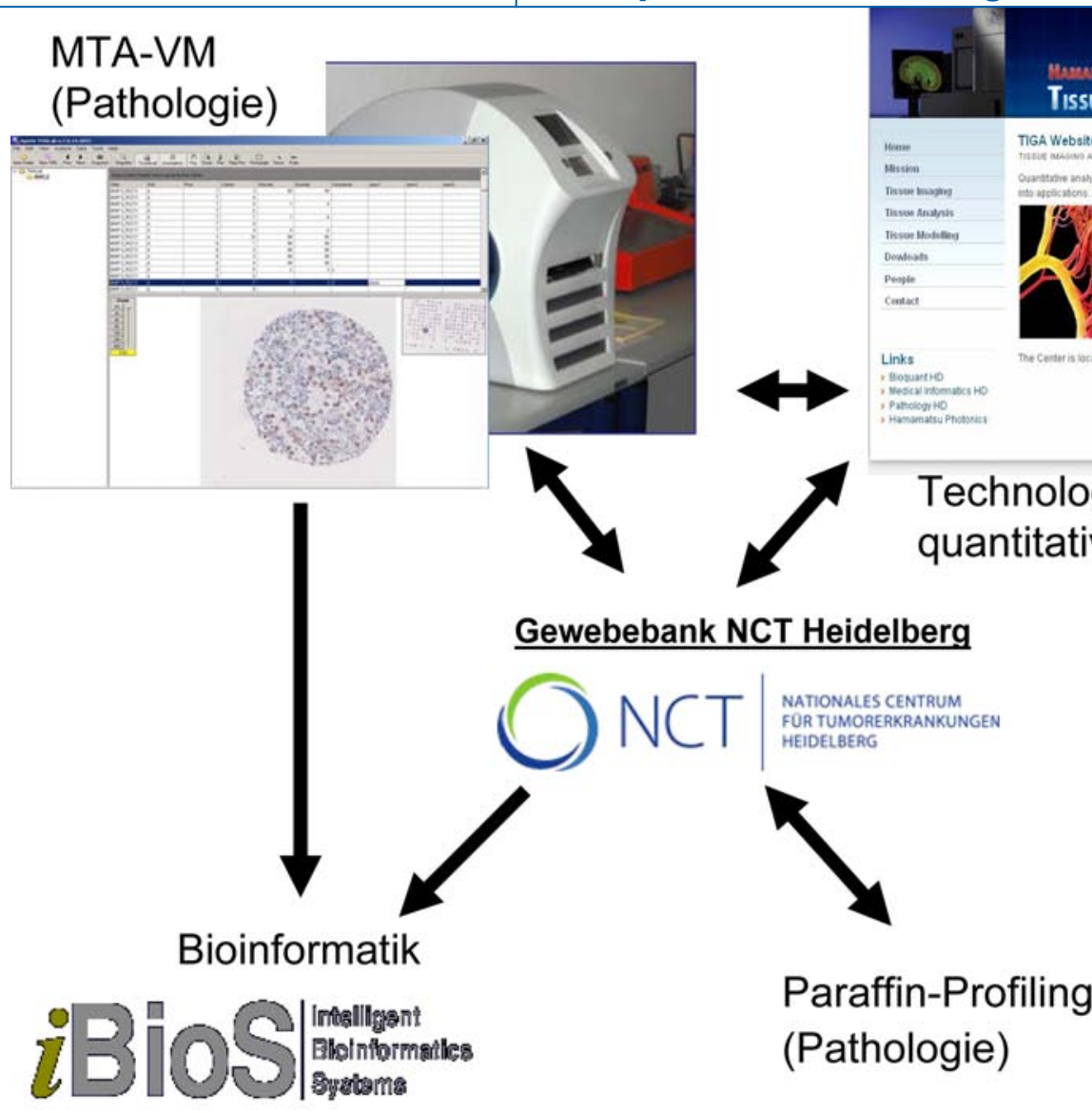

Abb. $2 \Delta$ Einsatz innovativer Technologien in der Gewebebank des NCT Heidelberg

Projekte mit interner Qualitätskontrolle können in der Regel gut auf diese Gewebe zurückgreifen.

- Paraffinblöcke und Schnittpräparate verbleiben aus klinischen/diagnostischen Gründen in der „Gewährsträgerschaft “ der Pathologie, können aber auf projektbezogene Anfrage der Gewebebank zur Verfügung gestellt werden.

- Bei vielen Projekten hat es sich bewährt, die Kollektive auf der Basis möglich vollständig erhobener Kliniks- und Verlaufsdaten zu erstellen (am besten Studien- oder studienartig erstellte Kollektive), um anschließende korrelative Analysen zu ermöglichen. Eine retrospektive Erhebung dieser Daten ist in der Regel ausgesprochen arbeitsintensiv, meist unvollständig und von einer Gewebebank selbst bei guter Ausstattung nicht zu leisten.
- Es bewährt sich die Einrichtung eines EDV-Systems in der Gewebebank, das mit den entsprechenden Informationssystemen der Klinik und der $\mathrm{Pa}$ thologie gut kompatibel ist und ggf. einen einfachen Datentransfer ermöglicht. Einen freien Zugang von Gewebebankdaten z. B. über ein Klinikinformationssystem halten wir aufgrund einer möglichen Interferenz mit dem Projektmanagement der Gewebebank nicht für erstrebenswert; es ist in Heidelberg nicht realisiert. Eine qualitativ hochwertige und entsprechend personell unterstützte Dokumentation ist für eine Gewebebank unverzichtbar.

- Für die vielfältigen technischen Anforderungen an eine Gewebebank (Paraffin- und Gefrierschnitte, Färbungen, MTAs, Immunfärbungen, ggf. Projektbetreuung) ist die Einrich-

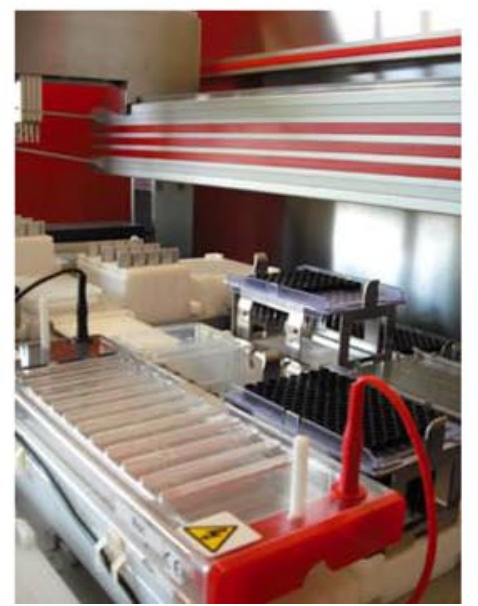

tung eines Gewebebanklabors sinnvoll.

\section{Ethisch-rechtliche Rahmenbedingungen}

Die gesamte Struktur (Geschäftsordnung, Aufbau, Entscheidungsprozesse einschließlich Ein- und Ausschlusskriterien für Projekte) wurde 2005 der Ethikkommission Heidelberg zur Prüfung vorgelegt. Nach umfangreicher Prüfung wurde das Gewebebankkonzept befürwortet. Das positive Votum der Ethikkommission beinhaltete:

- die Geschäftsordnung der Gewebebank,

- die einheitliche Einverständniserklärung zur Vorlage beim Patienten,

- die Entscheidungsfindung bei Projektanträgen,

- die Modalitäten der prospektiven Sammlung der Gewebe, 
- die Modalitäten bei Weitergabe der Gewebe und eventueller klinischer und histologischer Daten (Pseudonymisierung).

Im Jahr 2008 wurde nach Prüfung durch die Ethikkommission eine entsprechende Formulierung in den Patientenaufnahmevertrag des Universitätsklinikums übernommen. Dieser Passus ersetzt nicht die Einverständniserklärung, dient jedoch der zusätzlichen Erfassung des Patientenwillens und der weiteren Absicherung der Gewebebank. Unabhängig vom gewebebankbezogenen Votum existiert seit 2005 ein positives Votum der Ethikkommission Heidelberg, das die Verwendung von Geweben $\mathrm{ab} 3$ Jahren nach Entnahme ohne eine spezifische Zustimmung von Seiten des/der Patienten/in ermöglicht.

Das Ethikvotum zur Gewebebank wird auch zur Vereinfachung des Beantragungsprozesses bei wichtigen nationalen Forschungsförderungsorganisationen eingesetzt. Bei Projektanträgen an die Deutsche Forschungsgemeinschaft und die Deutsche Krebshilfe wird von beiden Organisationen seit 2006 akzeptiert, dass Antragsteller nicht einen eigenen Antrag an die Ethikkommission stellen müssen, sondern auf eine positive projektbezogene Stellungnahme der Gewebebank verweisen können. Dieses Vorgehen wurde bereits bei mehreren Projektanträgen des Standortes Heidelberg an die genannten Organisationen durchgeführt.

\section{Gewebebankassoziierte innovative Techniken}

Wesentlich für die Gewebebank Heidelberg war es, sie nicht nur als reine Ressourcen-, sondern auch als Technologieplattform zu entwickeln. Daher wurden und werden im Rahmen der Gewebebank verschiedene innovative Techniken vorgehalten und weiterentwickelt (• Abb. 2).

Eine zentrale gewebebankassoziierte Technologie ist die Multi-tissue-array (MTA)-Technologie. Sie ermöglicht es, in vielen Projekten schonend in Bezug auf die Menge des Ausgangsgewebes vorzugehen oder auch korrelative Expressionsanalysen oder Screening-Ansätze überhaupt erst mit vertretbarem Aufwand durchzuführen. Von entscheidender Be-

Pathologe 2008 · [Suppl 2] 29:204-209 DOI 10.1007/s00292-008-1067-2

(c) Springer Medizin Verlag 2008

\section{E. Herpel $\cdot$ N. Koleganova $\cdot$ P. Schirmacher \\ Gewebebank des Nationalen Zentrums für Tumorerkrankungen. Innovative Plattform für die translationale Tumorforschung}

\section{Zusammenfassung}

Die Gewebebank des Nationalen Zentrums für Tumorerkrankungen (NCT) Heidelberg wurde 2005 von der Universitätsklinik Heidelberg und dem Deutschen Krebsforschungszentrum als Sektion des NCT initiiert. Sie ist eine Non-Profit-Einrichtung mit einer komplett evaluierten ethisch-rechtlichen Rahmenstruktur und unterstützt die Prinzipien des Comprehensive Cancer Centers. Ziel ist sowohl die Gewinnung und Bereitstellung von frisch asservierten und paraffinfixierten humanen Gewebeproben für wissenschaftliche Projekte basierend auf den Prinzipien des "good scientific practice" als auch die standortumfassende projektbezogene Forschungsförderung. Projektunterstützung kann mittels eines kurzen Antrags bei der Gewebebank beantragt werden. Die Betreuung der Projekte erfolgt spezifisch durch er- fahrene Histopathologen und ggf. durch klinische Kooperationspartner.

Die Gewebebank des NCT stellt eine zentrale Plattform dar, welche gewebebankassoziierte, innovative Techniken wie z. B. „tissue-micro-arrays", virtuelle Mikroskopie, weiterentwickelt und Schnittstellen zur digitalen Bildverarbeitung sowie Bioinformatik schafft. In ihrer Verbindung von Ressourcen- und innovativer Technologieplattform besitzt die Gewebebank des NCT Modellcharakter im Bereich Biobanking und auch für Standorte mit einer aktiven, interdisziplinären Tumorforschung.

Schlüsselwörter Gewebebank · Comprehensive Cancer Center · Translationale Forschung · Good scientific practice - Ressourcen- und Technologieplattform

\section{Tissue bank of the National Centre for Tumour Disease. An innovative platform for translational tumour}

\section{Abstract}

The tissue bank of the National Centre for Tumour Diseases (NCT) in Heidelberg, Germany, was founded in 2005 by the University Hospital of Heidelberg and the German Cancer Research Centre as a section of the NCT. It is a nonprofit organization with a completely evaluated legal and ethical framework and supports the Comprehensive Cancer Centre concept. Its main aim is the acquisition and characterization of fresh-frozen and paraffin-embedded human tissues according to the standards of good scientific practice and the promotion of interdisciplinary tumour research of the comprehensive cancer centre and its cooperating partners. It also offers expert project assistance: a project leader can submit a short proposal, and the tissue col- lecting/preparing process will be performed in cooperation with a specialised pathologist and, if applicable, an experienced clinical researcher. The tissue bank is also a central platform for further developing of innovative technologies for tissue handling, e.g. multitissue-array and virtual microscopy, with links to digital image analysis and bioinformatics. Thus, the NCT tissue bank represents a model for innovative biobanking and for institutions with active interdisciplinary cancer research.

\section{Keywords}

Tissue banking - Comprehensive Cancer Center · Translational research · Good scientific practice $\cdot$ Resources and technology platform 
deutung ist hierbei, dass für die Erstellung der MTAs hinreichend große sowie komplett klinisch und histologisch charakterisierte Kollektive eingesetzt werden. Auch eine automatisierte Nukleinsäurenextraktion und -analyse lassen sich mit großem Nutzen mit der Gewebebank verbinden.

Weiterhin bietet es sich an, die Gewebebank eng mit der virtuellen Mikroskopie zu vernetzen, wie dies in Heidelberg seit 2006 erfolgt. Die virtuelle Mikroskopie erlaubt es zum einen, Schnittpräparate (z. B. Referenzschnitte) zu dokumentieren und jederzeit dem Projektleiter auch dezentral zur Einsicht zur Verfügung zu stellen, was besonders auch bei multilateralen Projekten eine zentrale Gewebebetreuung ungemein erleichtert (Plattform). Darüber hinaus ermöglicht die virtuelle Mikroskopie, MTA-Ergebnisse einfach zu erfassen und ebenfalls dezentralen Analysen zur Verfügung zu stellen. Hier ergeben sich Schnittstellen zur digitalen Bildverarbeitung. Die gescannten Präparate können ausgewertet werden und auch in entsprechende Dateiformate überführt und dort korrelativen Analysen unterzogen werden. Hierbei empfiehlt sich die enge Zusammenarbeit mit einer entsprechend ausgerichteten Bioinformatik-Einheit.

\section{Klinische und morphologische Daten als Grundlagen wissenschaftlicher Arbeit}

Wesentlicher Bestandteil einer interdisziplinären Zusammenarbeit ist die adäquate Akzeptanz aller wesentlichen Beiträge zum entsprechenden Forschungskonzept. In der Geschäftsordnung der Gewebebank Heidelberg ist ausdrücklich festgehalten, dass sowohl die Erhebung relevanter klinischer und bildgebender Daten als auch die konsequente Nachverfolgung der Patienten und die pathohistologische Charakterisierung eine entscheidende Grundlage jeder wissenschaftlichen Arbeit mit humanem Gewebe darstellen. Hierüber werden alle gewebeempfangenden Projektleiter informiert, und eine Verpflichtung zur angemessenen Berücksichtigung bei Anträgen und Publikationen wird von ihnen im Zusammenhang mit dem Übergabeprotokoll unterzeichnet. Zuwiderhandlungen können zum Ausschluss vom Beantragungsprozess führen. Dieser Aspekt wird jedoch von allen beteiligten Einrichtungen als zentral für die Akzeptanz einer Gewebebank angesehen und nach bisherigen Erfahrungen entsprechend akzeptiert. Besonders hervorzuheben ist auch, dass über diese Maßnahmen die Rolle der Gewebebank als Projektmakler, d. h. als sinnvolle Plattform für Projektvernetzungen (z. B. zwischen Gruppen der biomedizinischen Grundlagenforschung und klinischen Einheiten), gefördert wird.

\section{Qualitätsmanagement}

Um eine verlässliche und gleichbleibend hohe Qualität der Gewebebankarbeit im Sinne der Nutzer sicherzustellen, ist es unerlässlich, konsequente Qualitätsmanagementmaßnahmen zu implementieren. Diese umfassen verschiedene, in der Heidelberger Gewebebank realisierte strukturelle und auch wissenschaftliche Maßnahmen.

Wesentliche strukturelle Maßnahmen sind:

- der o.g. Aufbau der Gewebebank bzw. ihre Regularien und der aus ihnen resultierende interne Projekt-ReviewProzess,

- die Fixierung aller Gewebebankverfahren in Form von „standard operating procedures“ (SOPs); diese wurden in umfassender Form von 2005 bis 2007 in Heidelberg zusammengestellt,

- umfassende externe Reviews der Gewebebank; diese umfassen die regelmäßige (2-mal/Jahr) Berichterstattung der Gewebebankleitung an den Beirat der Gewebebank, den jährlichen Review der Gewebebank durch das NCT Heidelberg und den alle 3 Jahre erfolgenden externen Review im Rahmen der NCT-Begutachtung durch ein internationales Expertengremium,

- prinzipiell kann eine Gewebebank sowohl zertifiziert als auch (da sie eine Prüfleistung erbringt) akkreditiert werden; eine Akkreditierung strebt die Gewebebank Heidelberg für die mittelfristige Zukunft an.

Zentrale wissenschaftliche Qualitätsmanagementmaßnahmen sind:
- standardisierte Anträge an die Gewebebank, konsequente Prüfung und Bearbeitung aller Anträge nach wissenschaftlichen Grundsätzen durch die Gewebebank und Projektreviews durch den Beirat (bei Konfliktfällen),

- Sicherung einer möglichst optimalen Gewebequalität durch SOPs, standardisiert erfolgende histologische $\mathrm{Ge}$ webeausgangskontrollen und Erstellung standardisierter Gewebeübergabeprotokolle,

- regelmäßiges Projekttracking (in Heidelberg nach 90 und 180 Tagen) in Bezug auf Qualität und Verwendung des übergebenen Materials.

Internationale Erfahrungen zeigen, dass ein konsequentes Qualitätsmanagement auch von zunehmend entscheidender Bedeutung bei multinationalen klinischen Studien, Zulassungsfragen und Kooperation mit industriellen Partnern wird.

\section{Fazit}

Gewebebanken sind für die zukünftige Entwicklung der biomedizinischen Forschung einer der entscheidenden Taktgeber und absolut unverzichtbar. Der Zugang zu dieser wertvollen Ressource wird in Zukunft maßgeblich über den wissenschaftlichen und translationalen Erfolg entscheiden. Die Einrichtung einer innovativen, qualitätsgesicherten, effizienten und entsprechend ausgestatteten Gewebebank ist daher eine zentrale Aufgabe eines jeden Standortes, der in der biomedizinischen Forschung aktiv ist. Da die Gewebebank nur interdisziplinär erfolgreich sein kann, ist aufgrund der erforderlichen Hintergrunddaten, aber auch aus Akzeptanzgründen eine enge Einbindung der klinischen Einheiten, aus Gründen der Betreuung und der "good medical practice" eine direkte Anbindung an die Pathologie erforderlich. Realisierungskonzepte sind standortabhängig; die Einbindung der Gewebebank in ein CCC, wie sie in Heidelberg erfolgt ist, ist eine sinnvolle, alle o.g. Gesichtspunkte berücksichtigende Organisationsform, die ihrer Rolle als zentrale Ressourcen- und Technologieplattform gerecht wird. Die Gewebebank ist 
nicht nur ein wesentlicher Entwicklungsbereich der Pathologie, sondern bietet selbst eine ideale Plattform zur Applikation und Weiterentwicklung von innovativen gewebebezogenen Technologien (z. B. MTA, virtuelle Mikroskopie, digitale Bildverarbeitung) und Bioinformatik. Die Anforderungen an eine individuelle Gewebebank sind umfangreich und komplex und umfassen so unterschiedliche Bereiche wie ethisch-rechtliche Rahmenbedingungen, strukturelle Maßnahmen, wissenschaftliche Projektbetreuung, Qualitätsmanagement einschließlich umfangreicher "standard operating procedures" und technologische Weiterentwicklung. Diese vielfältigen Aufgaben erfordern eine ausreichende Ausstattung der Gewebebank, Diversifizierung ihrer Funktionen und auch intensive Kooperation der verschiedenen Gewebebanken untereinander.

\section{Korrespondenzadresse}

\section{Prof. Dr. P. Schirmacher}

Pathologisches Institut, Universitätsklinikum Im Neuenheimer Feld 220/221,

69120 Heidelberg

Peter.Schirmacher@med.uni-heidelberg.de

Interessenkonflikt. Peter Schirmacher ist Sprecher des Beirats der NCT-Gewebebank; Esther Herpel ist Leiterin der NCT-Gewebebank. 